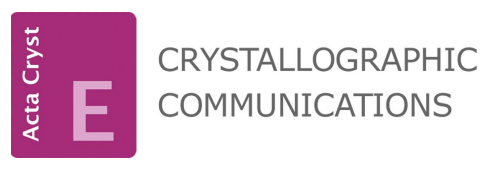

ISSN 2056-9890

Received 26 October 2018

Accepted 26 October 2018

Edited by D.-J. Xu, Zhejiang University (Yuquan Campus), China

\section{Crystal structure and Hirshfeld surface analysis of $(E)-N$-[(2-ethoxynaphthalen-1-yl)methylidene]- 5,6,7,8-tetrahydronaphthalen-1-amine. Corrigendum}

Sevgi Kansiz, ${ }^{a}$ Mustafa Macit, ${ }^{b}$ Necmi Dege ${ }^{a}$ and Galyna G. Tsapyuk ${ }^{c *}$

${ }^{\mathbf{a}}$ Department of Physics, Faculty of Arts and Sciences, Ondokuz Mayıs University, 55139, Samsun, Turkey, ${ }^{\mathbf{b}}$ Department of Chemistry, Faculty of Arts and Sciences, Ondokuz Mayıs University, 55139, Samsun, Turkey, and ${ }^{\mathbf{C}}$ Taras Shevchenko National University of Kyiv, Department of Chemistry, 64 Vladimirska Str., Kiev 01601, Ukraine. *Correspondence e-mail: tsapyuk@ukr.net

In the paper by Kansiz et al. [Acta Cryst. (2018), E74, 1513-1516], the address of the correspondence author is incorrect.

In the paper by Kansiz et al. (2018), the address of the correspondence author, Galyna G. Tsapyuk, should be 'Taras Shevchenko National University of Kyiv, Department of Chemistry, 64 Vladimirska Str., Kiev 01601, Ukraine', as given above.

\section{References}

Kansiz, S., Macit, M., Dege, N. \& Tsapyuk, G. G. (2018). Acta Cryst. E74, 1513-1516. 